\title{
Preventing Maternal and Pediatric Obesity by Optimizing Meal Properties
}

\author{
Akbar Nikkhah* \\ Department of Animal Sciences, Faculty of Agricultural Sciences, University of Zanjan, National Elite Foundation, Iran
}

*Corresponding author: Akbar Nikkhah, Chief Highly Distinguished Professor, Department of Animal Sciences, Faculty of Agricultural Sciences, University of Zanjan, Foremost Principal, Highly Distinguished Elite Scientist, National Elite Foundation, Iran, Tel: 0912-7891124; E-mail: anikkha@yahoo.com

Rec date: Nov 18, 2016; Acc date: Nov 19, 2016; Pub date: Nov 28, 2016

Citation: Nikkhah A. Preventing maternal and pediatric obesity by optimizing meal properties. Ped Health Res. $2016,1: 1$.

\section{Abstract}

Maternal obesity especially in visceral and abdominal regions is a rising problem that increases pediatric morbidity and reduces life quality. Minimizing the problem requires optimizing meal properties. This means optimizing meal size, frequency, timing and sequence. Frequent smaller meals evenly distributed over day-time with no large evening meals, and inclusion of fibrous plant meals in-between are an obligation for pre-gestating and pregnant women. These meal related strategies will allow the gut to maintain its healthiness towards optimal maternal and pediatric splanchnic and peripheral metabolism.

Keywords: Obesity; Meal; Pediatrics; Maternal; Health

\section{Discussion}

Maternal obesity is a rising concern in modern humans that reduces maternal and pediatric life quality and predisposes the body to a variety of complicated disorders. This reduces human life quality dramatically [1-3]. Thus, maternal interventions are needed to prevent the problem from happening. One significant area to work on is "meal properties". Meal properties include meal size, frequency, timing and sequence. Taking large infrequent meals by pregnant and lactating women is not considered healthy since it stimulates higher insulin peaks and increases adipogenesis, which in turn increases visceral adiposity. Instead, frequent small meals help the intermediary endocrinology to smoothen metabolism with no major shocks to the body. Maintaining a balance between substrate supply and use is key in preventing many maternal and pediatric complexities such as cancer [4-7].

Human has evolved to be active during day and inactive overnight. As such, human body is better prepared to assimilate nutrients during morning and day-time than evening and overnight $[3,8]$. This means that heavy food meals must not be taken overnight when the body cannot tolerate substrate overloads. Timing of meals, thus, needs to be optimized, which necessitates taking frequent meals during day and no large meal overnight [8-10].
Meal sequence requires increasing attention in today's modern and postmodern lifestyles. Frequent animal foods should be separated with frequent matching plant meals, supporting normal gut function and health with adequate fibrous (i.e. plant cell wall) materials. Such plant meals inhibit insulin shocks and help smoothen substrate absorption through the gut wall. In addition, plant and vegetable meals help successfully excrete fat residuals including cholesterol. Moreover, gut motility is maintained within healthy ranges, thus helping to reduce gut diseases with aging [11]. Timing of meals has already been suggested as a working strategy to optimize maternal and pediatric health [12].

Successful practice of meal optimization requires working on all meal properties. Thus, efforts must be made to optimize meal size, frequency, timing and sequence altogether and all the time to be able to observe positive outcomes.

\section{Conclusion}

Optimizing maternal meal properties involves taking frequent small meals evenly distributed over day-time with no large evening meals. This should be accompanied with plant meal intervals prepared from fibrous plant materials. Such a well-practiced formula should result in overcoming maternal and pediatric obesity related problems.

\section{Acknowledgments}

Thanks to the Ministry of Science Research and Technology, University of Zanjan and National Elite Foundation for supporting the author's global initiatives and programs of optimizing science edification in the third millennium.

\section{References}

1. Nikkhah A (2015) Evening exercise: A global strategy to prevent central adiposity and crdiometabolic diseases. Adv Weigh Manag Obes Cont. In Press.

2. Nikkhah A (2015) Breast safety progress through exercise-driven lactation: A pragmatic bioprocess to prevent cancer. J Bioprocess Biotech 5: e127.

3. Sehgal A (2004) Molecular biology of circadian rhythms. John Wiley \& Sons, Inc., Hoboken, NJ, USA. 
4. Nikkhah A (2015) Wrecked oncogenesis through synchronized substrate availability and oxidation: A novel bioengineering of cell physiology. Aust J Biotechnol Bioeng 2: 1042-1043.

5. Nikkhah A (2015) Nature as an ideal rhythm model for optimal cardiovascular physiology and health. Int J Diabetol Vasc Dis Res 3: $1-2$.

6. Nikkhah A (2015) Secure weight management via fitting circadian patterns of physical activity, resting and eating. Adv Weigh Manag Obes Cont 2: 23.

7. Nikkhah A (2015) Living on healthy rhythms to overcome cancer: Birth of a public therapeutic science. J Nutr Therap. In Press.

8. Nikkhah A (2015) Harmonizing eating and exercise circadian rhythms for optimal glucose-insulin and vascular physiology. Int J Diabetol Vasc Dis Res 3(3): 87-88.
9. Nikkhah A (2015) Lifestyle optimization: Today's foremost probiotic. J Probiotics Health 3: e119.

10. Nikkhah A (2015) Improving life quality via circadian timing of nutrient intake: Linking ruminant agriculture to human health. EC Agriculture 2: 258-259.

11. Nikkhah A (2016) Meal optimization to reduce obesity. Adv Obes Weight Manag Control, In Press.

12. Nikkhah A (2016) Maternal and pediatric obesity prevention by early dinner and late breakfast. Pediatrics Health Res, In Press. 